\title{
Status and First Results of the LHAASO Experiment
}

\author{
Huihai $\mathrm{He}^{1}$ for the LHAASO Collaboration* \\ Institute of High Energy Physics, CAS \\ Beijing 100049, China \\ E-mail: hhheihep.ac.cn
}

The Large High Altitude Air Shower Observatory (LHAASO) plans to build a hybrid extensive air shower (EAS) array with an area of about $1 \mathrm{~km}^{2}$ at an altitude of $4410 \mathrm{~m}$ a.s.l. in Sichuan province, China, aiming at very high energy gamma ray astronomy and cosmic ray physics around the spectrum knees. With an extensive air shower array covering an area of $1.3 \mathrm{~km}^{2}$ equipped with $>40,000 \mathrm{~m}^{2}$ muon detectors and $78,000 \mathrm{~m}^{2}$ water Cherenkov detector array, a sensitivity of about $1 \%$ Crab unit to gamma ray sources is achieved at $2 \times 10^{12} \mathrm{eV}$ and $5 \times 10^{13} \mathrm{eV}$, thus the LHAASO will survey the entire northern sky for gamma ray sources with full duty cycle and high sensitivity. The spectra of all sources in its field of view will be measured simultaneously over a wide energy range from from $10^{11} \mathrm{eV}$ to $10^{15} \mathrm{eV}$. This measurement will offer a great opportunity for identifying cosmic ray origins among the sources. The LHAASO is also equipped with 12 Cherenkov/fluorescence telescopes, so it will serve as an effective detector for energy spectrum measurement of different mass groups of cosmic rays over a wide energy range from $10^{14} \mathrm{eV}$ to $10^{18} \mathrm{eV}$.

Civil construction started in the middle of 2016, two years later, detector deployment began. The first water pool with 900 detector units covering an area of 22,500 $\mathrm{m}^{2}$ was put into operation in April of 2019. 1200 scintillation detectors, 300 muon detectors and 6 telescopes were deployed afterwards. 1/4 of the LHAASO array will be in operation soon. Preliminary results of the LHAASO experiment are presented.

36th International Cosmic Ray Conference -ICRC2019-

July 24th - August 1st, 2019

Madison, WI, U.S.A.

\footnotetext{
${ }^{1}$ Speaker

*for collaboration list see PoS(ICRC2019)1177

(C) Copyright owned by the author(s) under the terms of the Creative Commons 


\section{Introduction}

The Large High Altitude Air Shower Observatory (LHAASO) Huihai He for the LHAASO Collaboration, Radiation Detection Technology and Methods (2018) 2:7 plans to build a hybrid extensive air shower (EAS) array with an area of about $1.3 \mathrm{~km}^{2}$ at Mt. Haizi (4,410 m a.s.1., $29^{\circ} 21^{\prime} 27.6$ " N, 100 $08^{\prime} 19.6$ " E) in Sichuan province, China. With a sensitivity of about $1 \%$ Crab unit to gamma ray sources (Fig. 1), the LHAASO will survey the northern hemisphere for gamma ray sources with full duty cycle and a sensitivity of about $1 \%$ Crab unit to gamma ray sources at $2 \times 10^{12} \mathrm{eV}$ and $5 \times 10^{13} \mathrm{eV}$. The spectra of all sources in its field of view will be measured simultaneously over a wide energy range from $10^{11} \mathrm{eV}$ to $10^{15} \mathrm{eV}$. This measurement will offer a great opportunity for identifying cosmic ray sources among the gamma ray sources Y. Liu et al. for the LHAASO Collaboration, The Astrophysical Journal, 826:63 (8pp), 2016 July 20. Through hybrid detection of showers with the other detectors of LHAASO, the Cherenkov/fluorescence telescopes of LHAASO will effectively measure energy spectra of different mass groups of cosmic rays above $10^{14} \mathrm{eV}$ with an aperture of $>4000 \mathrm{~m}^{2} \mathrm{sr}$ Liqiao Yin et al. for the LHAASO Collaboration, Chinese Physics C, Vol. 43, No. 7 (2019) 075001, aiming for study of cosmic rays with energies in $10^{14}-10^{18} \mathrm{eV}$, where the cosmic ray spectrum knees around $10^{15.5} \mathrm{eV}$ and $10^{17.5} \mathrm{eV}$ are located.
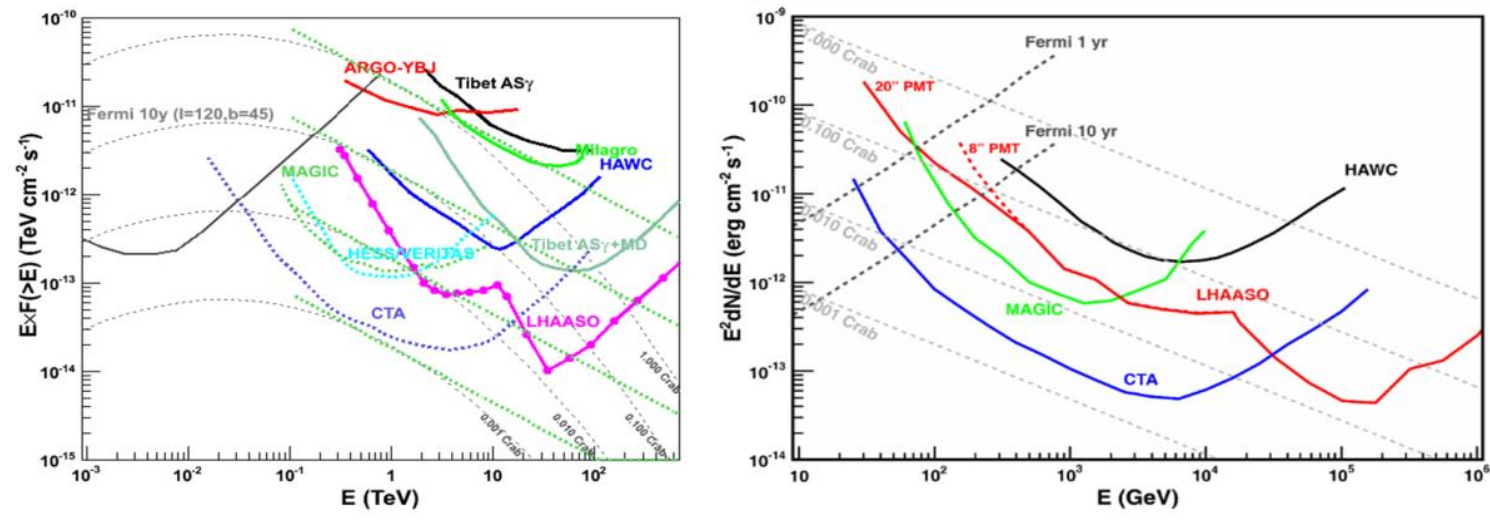

Fig. 1 LHAASO integral (right) and differential (right) sensitivity to gamma ray sources compared with other experiments/projects (1 year for Tibet $\mathrm{AS}_{\gamma}, \mathrm{ARGO}$-YBJ, HAWC and LHAASO, 50 hours for IACTs ) Abdo, A. A. et al. 2010, The Astrophysical Journal, 708, 1254 A.U. Abeysekara, et al., Astroparticle Physics 50-52 (2013) 26-32. The Crab spectrum energy distribution used here is got by fitting measurements of Fermi, ARGO-YBJ, Tibet AS $\gamma$,

MAGIC, VERITAS, HESS, HEGRA and Whipple.

The LHAASO consists of an extensive air shower (EAS) array covering an area of $1.3 \mathrm{~km}^{2}$ (KM2A) with 5195 electromagnetic particle detectors (EDs) and 1171 muon detectors (MDs), $78,000 \mathrm{~m}^{2}$ water Cherenkov detector array (WCDA) and 12 wide-field air Cherenkov/fluorescence telescopes (WFCTA), as shown in Fig.2. The total budget is about 1.2 billion CNY, and detector construction will last 4 years. For detector design and calibration, electronics, data acquisition, etc, see S. Cui et al. for the LHAASO Collaboration, Astroparticle Physics, 54 (2014) 86-92-B. Bartoli et al., Chinese Physics C, Vol. 38, 045001 (2014) for details. 


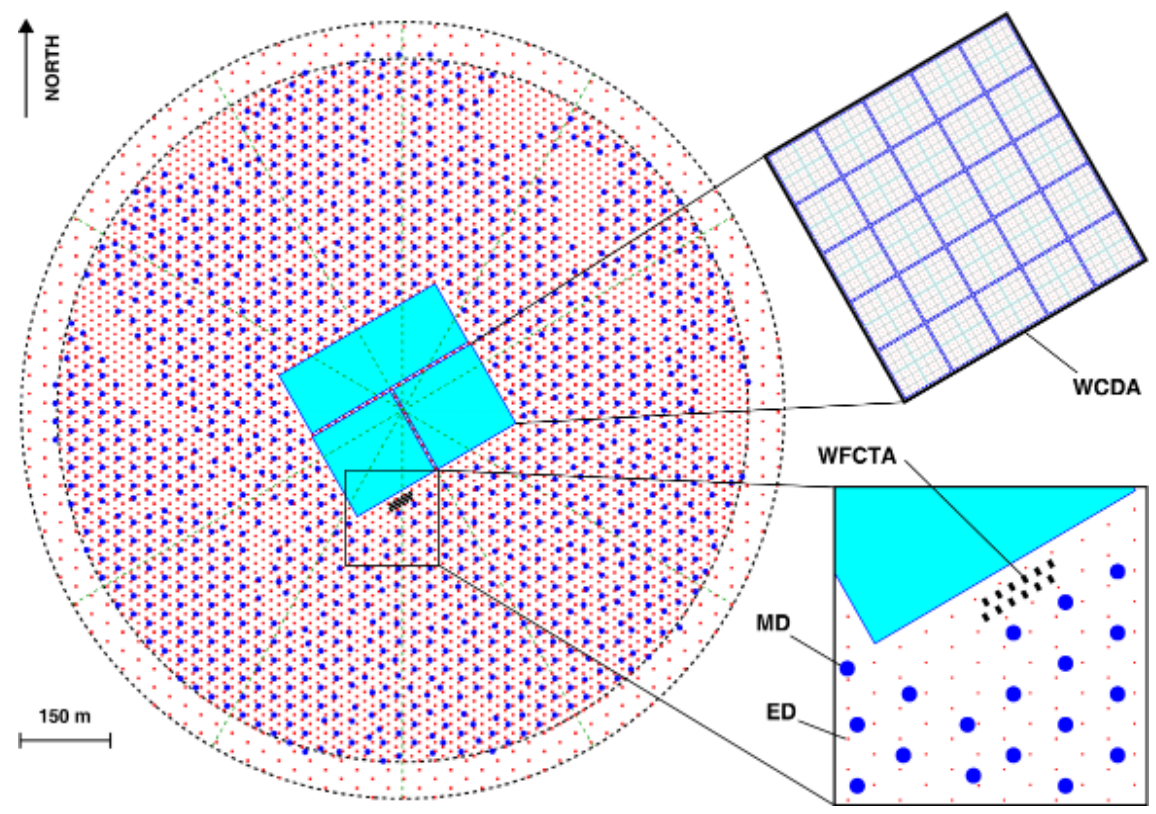

Fig. 2 Layout of the LHAASO experiment. The inserts show the details of one pond of the WCDA and of the KM2A array constituted by two over imposed arrays of EDs and of MDs.

WFCTA telescopes, located at the edge of a pond, are also shown.

\section{Status of the LHAASO Experiment}

Civil construction started in the middle of 2016. One year later, a $35 \mathrm{kV}$ power tranmission line was ready, climbing $29 \mathrm{~km}$ from an altitude of 3,800 m upto 4,410 m a.s.1.. In November 2017, the site was ready for construction of the water pools, and roads with a total length of about $20 \mathrm{~km}$ were built to reach each MD as shown in Fig.3-left.

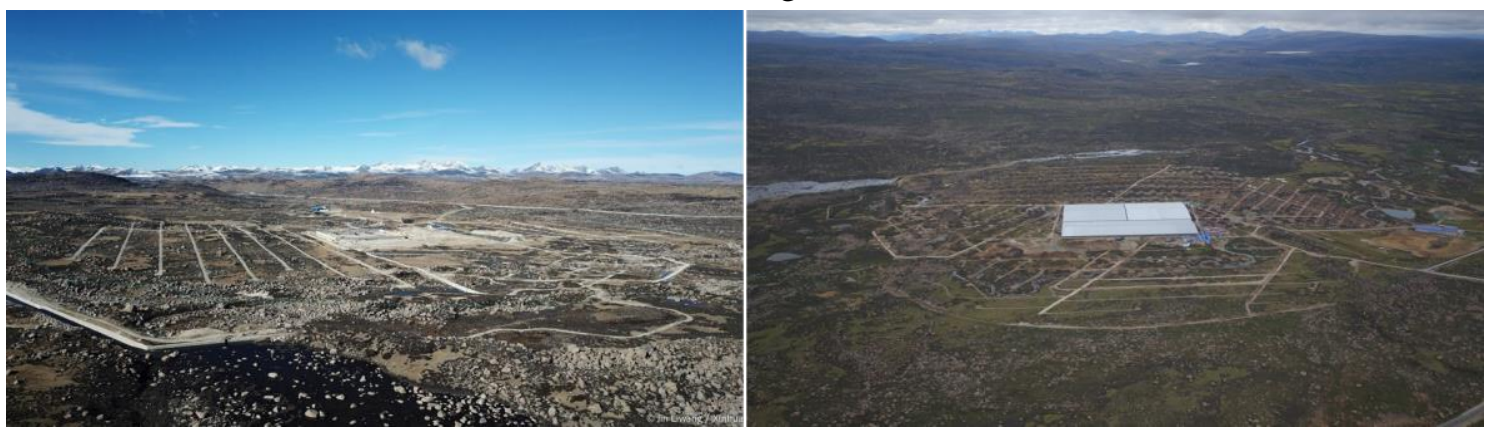

Fig.3 Photos of the LHAASO site in November 2017 (left) and in July 2019 (right).

\subsection{KM2A}

For field test, 33 EDs were deployed in January 2018 and have been in stable operation for about one and a half years (right part in Fig.4-left), another 33 EDs and 10 MDs, which are more than $200 \mathrm{~m}$ away from the first zone as shown by the left part in Fig.4-left, were deployed and added into the DAQ stream at the end of 2018. An event which fired all the EDs and MDs is shown in Fig.4. The number of muons detected by MDs as a function of the number of electromagnetic particles detected by EDs is in good agreement with MC simulation as shown in Fig.4-right. By using the data of the first 33 EDs, cosmic ray sun and moon shadows are observed as expected Hongkui $\mathrm{Lv}$ et al. for the LHAASO Collaboration, Offline calibration of electromagnetic particle detectors in LHAASO, ICRC2019. 
After field test, detector mass deployment started Xiaopeng Zhang for the LHAASO Collaboration, Batch measurement of attenuation length of WLS fibers for LHAASO-ED, ICRC2019-Lingyu Wang for the LHAASO Collaboration, Leak test for liner of muon detector of LHAASO, ICRC2019. Until now, 1200 EDs and 300 MDs have been deployed and will be added into the main DAQ stream soon.
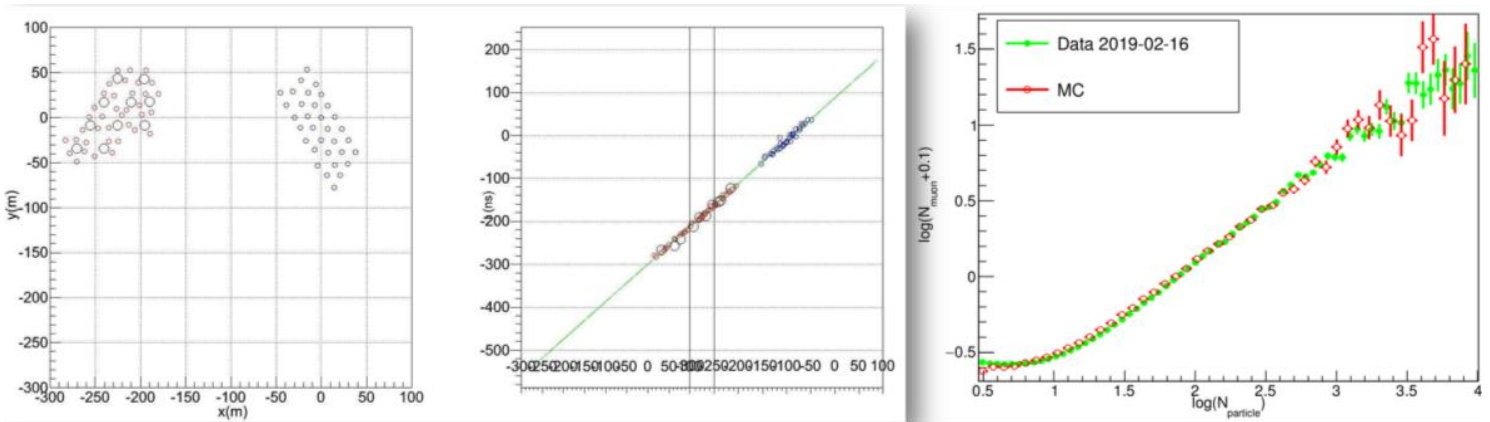

Fig.4 An event map (left) and its shower front (middle) are shown with 66 EDs (smaller circles) and $10 \mathrm{MDs}$ (larger circles) in operation. The right panel shows the profile of number of muons

detected by MDs vs. number of electromagnetic particles detected by EDs, MC simulation results are also shown for comparison.

\subsection{WCDA}

Construction of the first water pool was completed in Jan., 2018, and after one and a half years, the 3rd water pool is almost ready now (Fig.3-right). Detector deployment in the first water pool was carried out in 2018. Before the pool was filled with water (Fig.5-left), a so-called dry run was carried out, i.e. the PMTs acted as charged particle detectors. When a charged particle pass through PMT windows, it emmits Cherenkov photons if its velocity exceeds the speed of light in glass, thus the PMTs form a sampling EAS array. Through dry run, the PMTs, electronics, the data acquisition system, etc, were tested. The 1st pool was then filled with water and put into operation in April 2019 Mingjun Chen for the LHAASO Collaboration, Status and first result of LHAASO-WCDA, ICRC2019-Zongkang Zeng et al. for the LHAASO Collaboration, A charge calibration method for LHAASO WCDA dynamic range expansion system, ICRC2019.
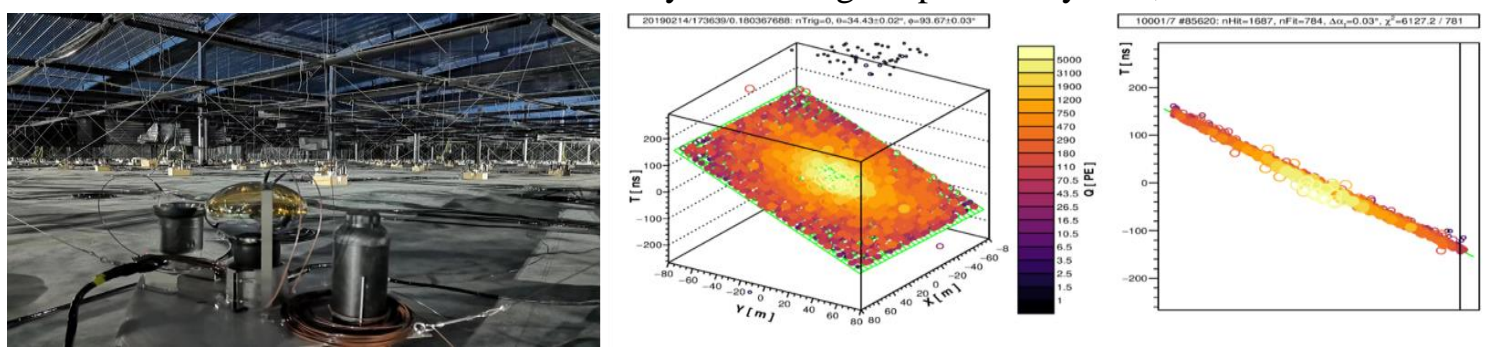

Fig.5 Photos of the 1st water pool before filled with water (left). The shower front of a huge event during dry run is shown in the right panel.

\subsection{WFCTA}

Six removable and adjustable containers with mirrors inside have been installed at the LHAASO site, 2 of them with SiPM camera installed are in operation. More than 220,000 EAS events coincident with the first WCDA pool have been collected with a typical event shown in Fig.6. See Shoushan Zhang et al. for the LHAASO Collaboration, Properties and performance of 
SiPM based-Mingjie Yang et al. for the LHAASO Collaboration, The performance of LED calibration system for Cherenkov telescope of LHAASO for details.
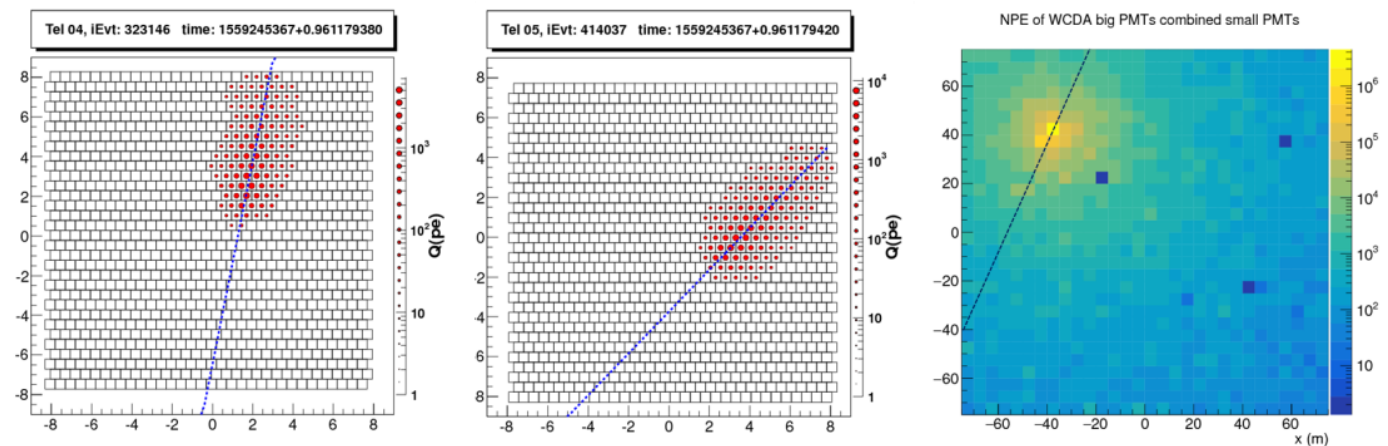

Fig.6. Images of a typical event seen by the first (left) and second (middle) telescopes located at the bottom-left corner of the first WCDA water pool, both pointing to the zenith with an azimuth distance of about 45 degrees. The blue curves in the figures are the major axises of the images, representing the shower detector planes. Map of the same event seen simultaneously by the 1 st

WCDA pool is shown in the right panel.

\section{Preliminary Results}

After three months' operation, about 30TB raw data were collected by the first WCDA pool, with a live time of about $95 \%$. The cosmic ray Moon and Sun shadows were observed with significances as expected.

By applying a Compactness cut on the data, almost 99\% of the backgroud events are rejected, while preserving $50 \%$ of gamma events with energies about $3 \mathrm{TeV}$ Xiaojie Wang et al. for the LHAASO Collaboration, Gamma Hadron separation using single parameter method and multivariate algorithms with LHAASO-WCDA experiment, ICRC2019. A full sky survey was performed on the data, more than 5 sources including Crab Nebula and Mrk 421 were detected with significances exceeding 5 sigma, as expected.

\section{Conclusions}

Constuction and detector deployment of the LHAASO experiment is going on smoothly. The first water Cherenkov detector array has been in data taking since April, 2019. Preliminary results show that the detectors are working as expected. A portion of KM2A with 300 MDs and 1,200 EDs have been deployed. 1/4 of LHAASO will start data taking soon. By the end of 2019, $1 / 2$ of LHAASO will be ready for operation. The whole array will be completed by the end of 2020.

It should be mentioned that, for the 2nd and 3rd WCDA water pools, 20 inch PMTs will be used to replace the 8 inch ones, and 3 inch ones to replace the 1.5 inch ones, to lower the threshold energy and to enchance the sensitivity at low energies.

\section{Acknowledgments}

This work is supported by the National Key R\&D Program of China (No. 2018YFA0404201 and 2018YFA0404202), the Chinese Academy of Sciences and the Key Laboratory of Particle Astrophysics, IHEP, CAS. 


\section{References}

[1] Huihai He for the LHAASO Collaboration, Radiation Detection Technology and Methods (2018) $2: 7$

[2] Y. Liu et al. for the LHAASO Collaboration, The Astrophysical Journal, 826:63 (8pp), 2016 July 20

[3] Liqiao Yin et al. for the LHAASO Collaboration, Chinese Physics C, Vol. 43, No. 7 (2019) 075001

[4] Abdo, A. A. et al. 2010, The Astrophysical Journal, 708, 1254

[5] J. Aleksic, et al., Astroparticle Physics 72 (2016) 76-94

[6] T. Hassan et al., Astroparticle Physics 93 (2017) 76-85

[7] K. Bernlöhr, et al., Astroparticle Physics 43 (2013) 171-188

[8] A.U. Abeysekara, et al., Astroparticle Physics 50-52 (2013) 26-32

[9] S. Cui et al. for the LHAASO Collaboration, Astroparticle Physics, 54 (2014) 86-92

[10] J. Zhao et al. for the LHAASO Collaboration, Chinese Physics C, Vol. 38, No. 3 (2014) 036002

[11] Z.Q. Zhang et al. for the LHAASO Collaboration, Nuclear Instruments and Methods in Physics Research, A 845 (2017) 429-433

[12] H.K. Lv et al. for the LHAASO Collaboration, Nuclear Instruments and Methods in Physics Research, A781 (2015) 34-38

[13] Chao Hou for the LHAASO Collaboration, Finalized design of LHAASO electromagnetic detector, ICRC2019

[14] Q. Du, G. Gong, W. Pan et al., Nuclear Instruments and Methods in Physics Research, A732 (2013) 488-492

[15] H. Li, G. Gong, W. Pan et al., IEEE TRANSACTIONS ON NUCLEAR SCIENCE, VOL. 62, NO. 3, JUNE 2015

[16] J. Liu et al. for the LHAASO Collaboration, Chinese Physics C, Vol. 38, No. 2 (2014) 026001

[17] X. Zuo et al. for the LHAASO Collaboration, Nuclear Instruments and Methods in Physics Research, A789 (2015) 143-149

[18] Zhou Bin, et al., Nuclear Instruments and Methods in Physics Research, A724 (2013) 12-19

[19] B. Gao et al. for the LHAASO Collaboration, Chinese Physics C, Vol.38, No.2 (2014)

[20] H. Li et al. for the LHAASO Collaboration, Chinese Physics C,Vol.38, No.1 (2014)

[21] Baomin Chen et al. for the LHAASO Collaboration, A study on the impact of the LHAASO-WCDA photomultiplier tube afterpulse, ICRC2019

[22] Liqiao Yin et al. for the LHAASO Collaboration, The expectation of cosmic ray proton and helium energy spectrum below 4 PeV measured by LHAASO, ICRC2019

[23] B. Bartoli et al., Chinese Physics C, Vol. 38, 045001 (2014)

[24] Hongkui Lv et al. for the LHAASO Collaboration, Offline calibration of electromagnetic particle detectors in LHAASO, ICRC2019 
[25] Xiaopeng Zhang for the LHAASO Collaboration, Batch measurement of attenuation length of WLS fibers for LHAASO-ED, ICRC2019

[26] Zheng Wang et al. for the LHAASO Collaboration, Performance monitoring of electromagnetic detectors in LHAASO, ICRC2019

[27] Lingyu Wang for the LHAASO Collaboration, Leak test for liner of muon detector of LHAASO, ICRC2019

[28] Mingjun Chen for the LHAASO Collaboration, Status and first result of LHAASO-WCDA, ICRC2019

[29] Zongkang Zeng et al. for the LHAASO Collaboration, A charge calibration method for LHAASO WCDA dynamic range expansion system, ICRC2019

[30] Xiaojie Wang et al. for the LHAASO Collaboration, Gamma Hadron separation using single parameter method and multivariate algorithms with LHAASO-WCDA experiment, ICRC2019

[31] Shoushan Zhang et al. for the LHAASO Collaboration, Properties and performance of SiPM based Cherenkov telescope for LHAASO, ICRC2019

[32] Mingjie Yang et al. for the LHAASO Collaboration, The performance of LED calibration system for Cherenkov telescope of LHAASO 